\title{
Filosofía de la gestión de la ciencia en Argentina a partir de la historia del CONICET
}

\author{
Philosophy of science management in Argentina from the history of CONICET
}

Elvio Galati (elviogalati@gmail.com) Centro de Altos Estudios en Ciencias Sociales, Universidad Abierta Interamericana (Rosario, Argentina)

\begin{abstract}
The methodology of this paper is documentary, compiling textbooks and scientific articles related to Argentine scientific history, mainly referring to CONICET. The purpose is to show that a complete epistemological study cannot be done without giving an account of its sociological and historical context, in this case, specifically referred to the management of science. The perspective of this paper is more philosophical than historical, doing discourse analysis and interpretation. Management, a sociological element, has a close relationship with science, and its study is part of epistemology. So, first, we must be careful to use the classification of basic and applied science in order to prioritize the latter with funding. Secondly, science should be autonomous but not independent from society and the market in which it is embedded, and the private sector should be harmonized with the public sector as regards scientific production. Third, betting on scientific production by Argentina helps demystify the classification of developed and underdeveloped countries, or the first and the third world. Fourth, in the management of science, university plays a key role, so management of university and the centralized management of the scientific bodies should be harmonized. Fifth, regarding the strengthening of the human component, subsidies offering should be balanced with the professionalization of research based on dedication.
\end{abstract}

Key words: epistemology, management of science, history of science, science law, complex thinking.

\section{Resumen}

La metodología de este ensayo es documental, recopilando libros y artículos científicos que hacen referencia a la historia científica argentina, principalmente referida al CONICET. El propósito es mostrar que no puede realizarse un completo estudio epistemológico sin dar cuenta de su contexto sociológico e histórico, en este caso, precisamente referido a la gestión de la ciencia. La perspectiva del trabajo es más filosófica que histórica, realizando análisis del discurso e interpretación. La gestión, elemento sociológico, tiene una relación estrecha con la ciencia, y su estudio forma parte de la epistemología. Por lo que, en primer lugar, hay que cuidarse de utilizar la clasificación de ciencia básica y aplicada para priorizar con financiamiento a la última. En segundo lugar, hay que autonomizar pero no independizar la ciencia de la sociedad y el mercado en los que está inmersa, así como armonizar la iniciativa privada con la pública en el ámbito de la producción científica. En tercer lugar, apostar a la producción científica por Argentina contribuye a desmitificar la clasificación entre países desarrollados y subdesarrollados, o del primer o tercer mundo. En cuarto lugar, en la gestión de la ciencia, juega un papel clave la universidad, por lo que habría que armonizar la gestión de la universidad y de los organismos centralizados en la administración 
de la producción científica. En quinto lugar, en relación al fortalecimiento del componente humano, debería equilibrarse el ofrecimiento de subsidios con la profesionalización de la investigación en función de la dedicación.

Palabras clave: epistemología, gestión de la ciencia, historia de la ciencia, derecho de la ciencia, pensamiento complejo.

\section{Introducción}

El estudio del Consejo Nacional de Investigaciones Científicas y Técnicas de Argentina (CONICET) con perspectiva histórica me dará la oportunidad de plantear algunas de las grandes preguntas que surgen a partir de la ciencia en su costado más sociológico, las cuales forman parte de la epistemología. Se contextualizará su nacimiento, haciendo referencia a algunos acontecimientos y hombres destacados en su desarrollo, preguntándome acerca de la gestión de la ciencia. Como dice César Lorenzano: "el historiador de la ciencia debe, en nuestros países, reflejar necesariamente aspectos de la ciencia practicada en ellos" (1995:6).

La metodología de este ensayo es documental, recopilando libros y artículos científicos que hacen referencia a la historia científica argentina, principalmente referida al Conicet. La perspectiva del trabajo es más filosófica que histórica, lo que justifica el material tomado como referencia para la reflexión. Se hará análisis del discurso e interpretación incluyendo en la epistemología las condiciones económicas, políticas y religiosas que influyen en la marcha de la ciencia. El marco teórico es histórico, el del pensamiento complejo, que comprende un conjunto de diversidades al interior de un mismo tema de estudio. En palabras de Edgar Morin, "la complejidad es la unión de la simplicidad y de la complejidad; es la unión de los procesos de simplificación que implican selección, jerarquización, separación, reducción, con los otros contra-procesos que implican la comunicación, la articulación de aquello que está disociado y distinguido; y es el escapar de la alternativa entre el pensamiento reductor que no ve más que los elementos y el pensamiento globalista que no ve más que el todo" (2005:144). El trabajo se estructura en una primera parte donde se tratan temas históricos y una segunda donde se tratan temas más bien relacionados con la historia reciente.

\section{El desarrollo histórico del CONICET}

El organismo, tal como lo concebimos hoy, no fue la primera institución que administró la ciencia en Argentina. Uno de los grupos que influyó en el desarrollo de sus comienzos fue la Asociación Argentina para el Progreso de las Ciencias (AAPC), la primera institución que agrupó a científicos con la idea de "promover un plan de acción transversal a los grupos disciplinarios con el objetivo de tener mayor capacidad de influir en las esferas de gobierno" (Orione 2008:21). Un nacimiento similar hubo en el Reino Unido, que recuerda la necesidad del agrupamiento entre los propios científicos: "La Real Sociedad toma nacimiento gracias al interés en las actividades científicas de sus miembros fundadores. El grupo de hombres apasionados de la ciencia que devendría la Real Sociedad tenía reuniones desde 1645" (Merton 2008:131). La ley 12338 que creó la AAPC, le dio dinero para que entregara becas a la investigación y subsidios. En relación a los modelos que se llevaron a cabo, uno de sus integrantes, Bernardo Houssay, trató de integrar la intervención del Estado con la ayuda de particulares.

La integración es clave en el pensamiento complejo. Así se emulaba a las asociaciones cooperativistas anglosajonas, que reunían aportes de "organismos del Estado con autarquía administrativa, con recursos 
propios, autorizados por ley para crear centros de investigación aplicada para programas específicos con la participación de la industria privada, el gobierno nacional o provincial, o las universidades" (Orione 2008:98). El grupo de Houssay no era afecto a la intromisión del Estado en el desarrollo de la ciencia, como tampoco a la vinculación estrecha entre ciencia e industria o producción, es decir, apuntaba a la promoción de la ciencia básica, mientras que en el primer gobierno del presidente argentino Juan Perón, la idea fue vincular la ciencia con el modelo productivo. En cuanto al premio Nobel, "su enfoque de la ciencia no conducía a que las investigaciones se plasmaran en el desarrollo del país; quizás porque aunque sostuviera que era indispensable aplicarla, y promover a partir de ella una tecnología propia, dejaba el proceso demasiado librado a la lógica espontánea de la investigación, pensando que eran una consecuencia inevitable de la ciencia básica" (Lorenzano 2013:3).

Cabe destacar el papel que cumplieron las investigaciones de Houssay en ciencia básica: "A partir de estos trabajos el equipo de Houssay logró comprender el papel de la hipófisis en los procesos metabólicos de carbohidratos y sustancialmente en la diabetes, un hallazgo que sirvió de base para el trabajo de otros investigadores acerca del rol desempeñado por las otras glándulas endócrinas" (Atrio 2006:48).

Por el contrario, "las actividades de ciencia y técnica fueron concebidas como un componente de la planificación económica. La consigna 'ciencia para el pueblo' fue el leimotiv que atravesó el ciclo de las dos presidencias de Perón" (Hurtado 2010:73). He aquí uno de las interrogantes que deben responderse: "una perspectiva utilitarista, concebida en clave más técnica que científica, orientada a la resolución de problemas locales específicos" (Hurtado 2010:74).

Lo que se distinguió de la AAPC, de la Asociación Física Argentina (AFA) y la Unión Matemática Argentina (UMA). Ya en 1951, el gobierno de Perón había creado el Consejo Nacional de Investigaciones Científicas y Técnicas (CNICyT). Tuvo a su cargo: "el diseño y ejecución del Primer Censo Científico Técnico Nacional. Era necesario conocer quiénes, con qué recursos, en qué lugares y cómo trabajaban en la tarea de investigación en nuestro país, tanto en el ámbito privado como en el público" (Atrio 2006:44).

El grupo de Rolando García, quien fue decano de la Facultad de Ciencias Exactas y Naturales de la Universidad de Buenos Aires y colega de Jean Piaget, consideraba que había que "vincular el desarrollo científico a un panorama más amplio de necesidades sociales y a un proyecto político más amplio. [...] el conocimiento científico era inscripto en un marco de aplicaciones industriales, desarrollo económico y necesidades sociales" (Atrio 2006:109). El mero hecho de la dependencia es algo cuestionable, no solo entre la ciencia y la sociedad. Si la ciencia depende de la sociedad, se mimetiza con ella y poco puede contribuir a cambiarla.

Creado en 1958 por el gobierno de facto, a instancias del vicepresidente Isaac Rojas, tomó el CONICET los bienes y personal de la Dirección Nacional de Investigaciones Científicas y Técnicas (DNICyT) creada en 1950. Respondiendo a una de las grandes preguntas de la gestión de la ciencia, en definitiva a una cuestión epistemológica, el grupo incipiente creía que "la ciencia debía financiarse, pero debía ser liberada de las intervenciones ajenas a su propia lógica interna" (Hurtado 2010:109). En la misma línea de pensamiento, Houssay deja librada la ciencia a las iniciativas espontáneas, ejemplares, de los investigadores, ahuyentando la planificación: "quien se niega a cualquier planificación de la ciencia -pensando que equivalía a sovietizarla-, y propone que sean los científicos individuales los que propongan proyectos y soliciten fondos" (Lorenzano 2013:4). 
Otra disputa que aun hoy subsiste se dio a propósito del desarrollo de las Ciencias Sociales. Recuérdese que ya en el comienzo de la planificación de la ciencia y los organismos consecuentes que aparecían, en el caso de Houssay y la AAPC, no solo él era fisiólogo, sino que estuvo compuesta por mayoría de médicos y químicos. Hay que destacar que el anteproyecto de creación del CONICET fue presentado por la Academia Nacional de Ciencias Exactas, Físicas y Naturales, para luego convertirse en el decreto ley 1291/58 (Charreau 2008:261). En cuanto al fomento de la investigación, en el período 1935-1946, de 40 becas externas, 12 fueron a médicos, 12 a ingenieros y 8 a químicos y bioquímicos, de 6 becas privadas, todas fueron a médicos, y de 45 becas internas, el $60 \%$ fue otorgado a médicos. En relación a los subsidios, de 87, un $30 \%$ fueron a médicos y un 30\% a químicos y bioquímicos (Hurtado 2010:39). "La distribución de los fondos por área de conocimiento muestra que la tendencia a favor de las áreas biomédicas, del período 1858/9, se mantendría en los años siguientes hasta 1966" (Atrio 2006:46).

Luego sería García quien disputaría con Houssay acerca de la necesidad de que las ciencias sociales cuenten con becas. Con el retorno a la democracia se promovió la incorporación de investigadores en el ámbito de las ciencias sociales. El derecho de la ciencia y la tecnología, nacido para proteger al débil, como ocurre en todas las ramas jurídicas, debería hacer especial hincapié en la protección del cientista social, históricamente discriminado. Las ramas jurídicas también surgen "para evitar excesos concretos contra ciertas personas, como en el Derecho del Trabajo" (Ciuro Caldani 1992:851).

La creación de la carrera del investigador científico para el CONICET en 1961 da la pauta de la profesionalización que implica la investigación y la importancia que se le asigna, como cuando recién en 2013 la Facultad de Derecho de la Universidad Nacional de Rosario, en convenio con la Escuela de Educación de la Facultad de Humanidades y Artes de la misma universidad, implementan un convenio para desarrollar el "Profesorado en Derecho", cuando en universidades privadas ya existía antes de 2003. Lo que implica jerarquizar la actividad docente, distinguiéndola de la mera profesión de base.

La creación de un organismo especializado para la administración de la ciencia y de la carrera del investigador no puede soslayar la necesidad de asociaciones como la AAPC, en tanto es necesario bregar por organismos que defiendan al investigador científico. Por ejemplo, ante negativas arbitrarias de ingresos, promociones, formalización de situaciones precarias como las de los becarios, que se les exige como empleados, pero que no forman parte de la planta permanente y son fácilmente domesticables o especialmente vulnerables. Recién en el año 1973 se dio la esperada jerarquización al personal de la investigación científica: "ya no es el CONICET de Houssay, que tenía una carrera como nomenclatura de categorías para dar un adicional a los profesores universitarios que hacían investigación" (Hurtado 2010:135). Frente a las creencias que señalan la postergación del presupuesto en ciencia y los recortes que impactan en la apertura de fondos para el ingreso a carrera del investigador o los destinados a becas, se puede leer un pronunciamiento de la Asociación de Física Argentina en 1997, a propósito de un proyecto de instalación del Observatorio Pierre Auger para la detección de rayos cósmicos, liderado por un premio Nobel en Física, para el cual se pedía el aval de la AFA ante un hecho ya consumado: "no resultaba evidente si era 'más provechoso que la Secretaría de Ciencia y Técnica destine 15.000 .000 de dólares a un único proyecto de física o los destine a incorporar un mayor número de jóvenes investigadores postergados, o a aumentar el número de becas'" (Hurtado 2010:204). Plata siempre hay, poca o mucha, y lo que hay que pensar es a qué destinarla.

Una actitud que merece análisis es la del directorio del CONICET a raíz del golpe militar a Arturo Illia: "sus actividades -eminentemente técnicas- ni por la modalidad del nombramiento de sus miembros -por elección interna, luego dispuesta por decreto del Poder Ejecutivo con un mandato por tres años- deben 
los directores presentar sus renuncias. [...] se trata de un grupo de científicos que trabaja independientemente de toda consideración política" (Hurtado 2010:128). Esto significa que la ciencia solamente se guía por su lógica interna, es decir, por lo que se conoce como contexto de justificación, sin inmutarse ante los avatares políticos o sociológicos. El hecho es aún más cuestionable cuando el gobierno plantea la intervención a las universidades, derivándose en la conocida "Noche de los Bastones Largos". Una puesta a prueba de la relación entre política y ciencia se ha dado cuando regímenes de facto desplazaron a científicos y los que quedaban se debatían entre la renuncia o la permanencia. Puede tomarse la renuncia como una manera de adscribir la ciencia al científico, lo que a su vez se relaciona con el hecho de encarnarla en la realidad y no dejarla en lo abstracto de los bienes o conceptos: "Para ellos, la ciencia era la información que puede ser guardada en gráficos, cuadros y textos, no el conocimiento que sólo puede ser atesorado en la cabeza humana", según lo piensa Marcelino Cereijido (Orione 2008:47).

Con la creación del CONACYT (Consejo Nacional de Ciencia y Técnica) en 1969, durante el gobierno de Juan Carlos Onganía, se planteó el hecho de la división entre quienes formulan la política científica y quienes hacen investigaciones. La instauración del organismo fue sugerida al presidente cuando no fue posible sacar del cargo a Houssay y entonces pasó a ser un Ministerio de Ciencia que interfería en la actividad de todos los organismos que promovían e investigaban científicamente.

Durante el período 1972-1975, el presupuesto asignado al CONICET representó el 11,4\% de las inversiones públicas en ciencia y tecnología, mientras que durante el gobierno de facto pasó al $23 \%$. Lo cual guardaba relación con la persecución y desintegración que sufrieron las universidades durante el llamado "Proceso de Reorganización Nacional" (Hurtado 2010:149). No obstante, las consecuencias negativas de los años de plomo se esparcieron también a la actividad científica: "Durante los años de la dictadura, las transformaciones en las modalidades operativas del Conicet se completaron con irregularidades en el manejo de una parte importante de subsidios a través de fundaciones privadas que desviaron recursos para fines propios, que no tenían relación con la investigación científica" (Abeledo 2009:76).

En la década de los 90, con la administración de Carlos Menem, el ingreso a la carrera estuvo muy restringido, justamente por la visión economicista que tenía de la vida en general. En efecto, "la consigna de 'achicamiento del Estado' significó en el área de ciencia y tecnología la reducción del tamaño de las instituciones, es decir, de sus recursos materiales y, principalmente, de su personal" (Hurtado 2010:189). Siempre la ciencia va detrás de la economía: "en 1994, Cavallo [Ministro de Economía de Menem] impulsaba [...] el achicamiento de la poca ciencia que ya quedaba en el país" (Orione 2008:10). La reducción del apoyo del Estado se vio en el ámbito científico y también en el tecnológico: "En 1990, en el contexto de 'racionalización' de la administración nacional, el INTA cerró las puertas el 30 de diciembre y las volvió a abrir el $1^{\circ}$ de febrero con el 30\% del personal" (Hurtado 2010:191). En 1997 se reclamaba: "poner en marcha los ingresos y las promociones en la carrera del investigador [...] [y] asignar recursos para hacer frente a gastos de investigación. Ambas medidas estuvieron suspendidas por años, lo que generó malestar y desaliento profundos en el mundo académico" (Ciencia Hoy 1997:1).

El dinero tiene un valor extra además del poder adquisitivo y tiene que ver con el poder y el reconocimiento. En este sentido, durante los gobiernos de Néstor Kirchner y de Cristina Fernández, se produjo una relación inversa a lo ocurrido durante el gobierno de Menem, es decir, el Conicet tuvo salarios mayores a los de la Universidad. Como el Estado está siempre presente, aunque se lo achique, hay que tener en cuenta que en 1999 se denunciaba a: "Ios que fuerzan el cambio de director de trabajo en el caso de las becas postdoctorales. Este criterio atenta contra la libertad de elección temática de los becarios y 
acarrea el desmembramiento de grupos de trabajos consolidados, a quienes el CONICET debería apoyar sin retaceos" (Adler 1999:106).

Otro signo del corrimiento hacia la empresa y la extranjerización fue la ley de Promoción y Fomento de la Innovación Tecnológica, que en los hechos significó una protección a la propiedad intelectual que se generaba a raíz de la ciencia que se producía en otros lugares y que en la práctica se traducía en el pago de regalías por su uso. Hay que agregar también que el contexto político-global en el que las autoridades inscribían a Argentina era promoverla como exportadora de materias primas. Esta línea de acción tenía sus antecedentes cuando Uriburu sometió las actividades científico-técnicas a los intereses norteamericanos, cuando se cerró el proyecto Cóndor y se intentó privatizar la CNEA (Comisión Nacional de Energía Atómica) y otras instituciones de investigación por parte del gobierno menemista (Orione 2008:41).

En ese período se ve una profunda adhesión a la idea de que la ciencia y la tecnología no les corresponden, como don, a países periféricos como Argentina. Así, "todas las partes del Cóndor II desarrolladas en la Argentina fueron llevadas a Estados Unidos, vía España, para la supervisión de su destrucción. [...] el desarrollo espacial en la Argentina volvía a punto cero" (Hurtado 2010:200). En el caso del gobierno argentino posterior a la primera presidencia de Juan Perón, "la nueva red de actores que se comenzó a conformar alrededor del desarrollo aeronáutico fue proclive a la compra de aviones caza en el extranjero" (Hurtado 2010:103). Esto indica que la ciencia aeronáutica argentina no entraba en los planes de desarrollo. Al contrario, el gobierno peronista desarrolló la investigación en ciencia y técnica vinculada con la energía nuclear y la aeronáutica, lo que manifiesta la intención del gobierno de promover la ciencia en dos ámbitos muy sensibles.

A pesar del aliento a la investigación, la repatriación de científicos y la creación de un Ministerio de Ciencia, Tecnología e Innovación Productiva, lo cierto es que en gran medida la ciencia es reconocida por el presupuesto que se le asigna y el personal humano que la integra, es decir, sus científicos: "este proceso de reconstrucción comenzó con Néstor Kirchner, que fue el primer presidente que se preocupó genuinamente por mejorar la condición de los investigadores" (Barañao 2012:340). No obstante, luego de 2011, con recortes en el ingreso y salarios asfixiados por la inflación, es necesario aumentar la planta de calidad. "[...] sin una inversión genuina, sin salarios adecuados, no hay ciencia de calidad" (Barañao 2012:341). Ya se dijo: "el concepto vertido por el Dr. Bernardo Houssay, en el sentido que la ciencia no tiene fronteras pero los científicos tienen patria, debería estar acompañado por condiciones tales que evitasen que becarios calificados queden fuera del sistema porque el presupuesto del CONICET, o la rigidez de su planta, no permiten su incorporación en la Carrera del Investigador" (Adler 1999:106).

El Ministro de Educación, Ciencia y Tecnología de Néstor Kirchner fue Daniel Filmus. Durante ese gobierno, el presidente del CONICET decía: "invertir en conocimiento implica levantar los ojos del piso de las coyunturas para fijar la mirada en el horizonte del desarrollo de nuestra nación y el bienestar de las próximas generaciones" (Atrio 2006). Evidentemente, no se hacía referencia al inmediatismo de la vinculación entre conocimiento y su aplicación o la economía de un país.

\section{La historia reciente de la ciencia argentina}

Llamo historia reciente a los hechos ocurridos a partir de la segunda presidencia de Cristina Fernández de Kirchner en 2011 y hasta su culminación el 10 de diciembre de 2015. 
Las típicas preguntas sobre qué, cómo, cuándo, dónde y quiénes se dan también en el ámbito de la gestión de la ciencia. Cabe preguntarse qué problemas investigar y por quiénes. Dos actores que tienen relación y protagonismo con la producción científica son el Estado y los organismos privados, como empresas. Frente a ello, se ha dicho que como las empresas se ocupan de los problemas inmediatos, que generan ganancia, más urgentes y locales, el Estado debe "dedicarse a tareas de mayor complejidad y de mediano y largo plazo, es decir, al tipo de problemas que no resultaban rentables a corto plazo para el sector privado" (Hurtado 2010:100). La pregunta por el objeto lleva inmediatamente a la pregunta acerca de cuándo y quiénes, e involucra al "cómo", ya que la financiación pública o privada influye en la cantidad y calidad de los resultados. En el cómo hay que preguntarse si la ciencia va a desarrollar un modelo propio o va a tomar sugerencias de extranjeros o la imitación.

Para dar libertad al científico y ser leal a él, habría que preguntar por quienes tengan vocación por la aplicación o ciencia aplicada o desarrollos tecnológicos. De lo contrario, se corre el riesgo de autorizar o financiar las investigaciones básicas que estén en afinidad con el gobierno de turno, si la mayor parte del presupuesto se vuelca a la ciencia aplicada: "lo único para lo que ha servido en el pasado [la clasificación entre ciencia básica y aplicada] entre nosotros es para justificar la reducción en el apoyo oficial a la primera, porque no estaba dirigida a resolver los 'problemas nacionales' o porque no caía dentro de las 'prioridades' establecidas en ese sexenio" (Pérez-Tamayo 2001:370).

A favor de la "ciencia básica" se ha hecho hincapié en "el error de adoptar y absorber las aplicaciones de la ciencia antes que la ciencia misma, y en no advertir que detrás del excitante esplendor del progreso industrial y técnico se oculta ese trabajo puro y desinteresado que en gran medida ha contribuido a aquel progreso material" (Orione 2008:25).

Una de las grandes preguntas que se realizan las instituciones es cómo producir conocimiento científico. Para responder a esa pregunta, las respuestas que se brindan son, encarando desde el Estado un organismo que centralice la administración de la ciencia, como ocurre en la Europa continental, en los casos de Francia, Italia, España, o dejando a la libre iniciativa de los individuos y sociedades, como ocurre en el Reino Unido y EE.UU. Otra pregunta es si integrar la ciencia al sector productivo, por ejemplo, apuntando a resolver problemas relevantes para la sociedad, atender las demandas del mercado o autonomizando la ciencia de la sociedad y el mercado, para que no se uniformice el pensamiento de un país.

Lo que tienen en común la actividad empresarial y la actividad científica es la iniciativa, el riesgo, las ganas de producir más. Características hoy puestas en duda en el escenario argentino ante su insuficiente pujanza industrial, tecnológica y científica, comparando con las potencias mundiales. Subrayaría de todas las formas posibles la palabra "producir", lo que implica trabajo, voluntad, ganas de superación y el reconocimiento al más alto nivel de esos resultados.

Tal vez no se hayan podido integrar dos modelos que parecen antagónicos: "un régimen de acumulación que intentó impulsar la industrialización por sustitución de importaciones orientada al mercado interno a otro régimen de apertura de la economía, desregulación de los mercados y desindustrialización" (Hurtado 2010:18). La gran influencia de la economía a escala global también se percibe a nivel científico cuando en el escenario global se habla de la división internacional del trabajo, donde tradicionalmente, a la clasificación de "primer mundo" y "tercer mundo" se asocian las ideas de "países desarrollados" y "países subdesarrollados" o "países en vías de desarrollo", naturalizándose la idea de que cada cual tiene su posición en el escenario mundial. La escasa apuesta económica y cultural a la ciencia tiene su consecuencia 
en la conservación de dicho estado de situación. Nótese que durante mucho tiempo se ha visto a la Argentina como el "granero del mundo".

Cuando se apunta a planificar la investigación científica se derivan ventajas y desventajas. Entre las ventajas, se asiste a la posibilidad de evaluar el proyecto, sea desde el principio, al decidir financiarlo, al momento de ver su implementación, si es como se le esperaba, y en sus resultados, para cambiar lo que haya que cambiar. En la planificación se enmarca el control y evaluación de las actividades de los científicos, por ejemplo: "se carece de información censal nacional sobre los resultados de estos proyectos en publicaciones, patentes o innovaciones tecnológicas" (Orione 2008:80). En febrero de 2014 pensaba que no habría estado mal que el CONICET organice y administre un repositorio digital de los productos de sus investigadores, lo que recién se dio en setiembre de 2015 (ri.conicet.gov.ar), además de financiar la publicación, por ejemplo, de las tesis doctorales o informes posdoctorales, ya que algunas editoriales suelen aplicar a las ciencias sociales criterios muy mercantilistas a la hora de exigir la venta de ejemplares. Además, para algo tiene que servir que algunas tesis tengan la recomendación de publicación, como mención especial que hacen los jurados doctorales. Como desventaja de la planificación, se puede permitir una excesiva burocratización de la empresa científica, ahogando iniciativas útiles que no cumplen con algún requerimiento o que no superan los obstáculos del caso o corrupción que pueda imperar.

En el marco de la historia argentina, hay que pensar el lugar de la universidad en relación con la ciencia. Sobre todo teniendo en cuenta a los científicos que eran separados de sus cargos en las universidades por diferencias ideológicas o políticas. Por ejemplo, durante el proceso de creación del Instituto Nacional de Tecnología Agropecuaria (INTA) se temía que monopolizara la experimentación agropecuaria, relegando las actividades de investigación que se hacían en las universidades (Hurtado 2010:97).

También la universidad presenta el problema de si su misión incluye a la investigación científica. En efecto, "siempre trabajó [Houssay] con el convencimiento que las universidades debían ser principalmente centros de investigación científica, siendo el ámbito óptimo para crear y propagar el conocimiento" (Atrio 2006:47). A pesar de todo, ubicar a la universidad como un lugar de ciencia no solo es valioso en sí, sino en relación con alti-bajos que puedan experimentarse en organismos que centralicen la administración de la ciencia como el CONICET, que ha tenido momentos de desfinanciación y que antes de su nacimiento tomó forma en distintos organismos que cambiaban frecuentemente el lugar de la ciencia según la perspectiva de los políticos de turno. Véase por ejemplo el caso de los 90, en donde la universidad cobró un papel importante en ciencia y tecnología ante el desfinanciamiento del CONICET. La universidad contribuye a paliar los déficits infraestructurales de la investigación argentina. Hoy mismo se dice: "el Conicet no puede crecer mucho más rápido que eso porque por más que hagamos un plan de infraestructura acelerado, no tenemos dónde meterlo" (Barañao 2012:348).

Al conectar el Estado con la ciencia, hay que pensar en su financiamiento, lo que se relaciona con la dedicación del científico. Un sistema alternativo a la profesionalización y/o a la dedicación exclusiva es el del subsidio a la investigación: "Los investigadores con sitio de trabajo en la universidad o en institutos privados se presentan de forma espontánea al CONACYT [de México] con declaración de dedicación exclusiva, producción científica en los dos últimos años y proyecto de investigación. Las solicitudes deben renovarse cada dos años y son juzgadas por comisiones ad hoc según la especialidad" (Dosne Pasqualini 2008:313).

Este sistema guarda alguna afinidad con lo que se conoce en Argentina como un "estímulo" a los docentes que hacen investigación a través del Programa de Incentivo a los Docentes-Investigadores. Esta fue la idea 
de Dante Caputo, Secretario de Ciencia y Tecnología del ex presidente Fernando De La Rúa: "los nuevos miembros de la Carrera del Investigador del CONICET deberían obtener un puesto en alguna universidad y, a cambio, recibirían del CONICET un suplemento de dinero sobre su salario universitario" (Hurtado 2010:211). Lo que iba contra la profesionalización del investigador: "Muchos interpretaron que esta iniciativa destruiría la estructura de la Carrera del Investigador. La rápida reacción de la comunidad científica hizo que este plan no pudiera ser implementado" (Hurtado 2010:212). Debería equilibrarse el ofrecimiento de subsidios con la profesionalización de la investigación en función de la dedicación.

Otra manera fue dejar a la iniciativa privada, a la organización espontánea, la empresa científica: "La creación de una universidad libre basada en institutos de investigación debe ser la obra de los industriales, los ganaderos, los agricultores, los comerciantes, los viticultores, los cañeros, en una palabra, de las llamadas fuerzas vivas del país. [...] su papel consiste en crear riqueza [...] verán a un estado burocrático absorber poco a poco todas las actividades que legítimamente les corresponden" (Hurtado 2010:56).

Esto que señalaban algunos investigadores en 1940 como proyecto, rememora las ideas del conde de Saint-Simon, que instauró la clásica distinción entre útiles e inútiles, es decir, los que producían riqueza y los que vivían de ella, para culminar en la administración de las cosas por parte de aquellos que producían. La aplicación de estas ideas significaría que la ciencia quede en manos de la iniciativa privada, guiada por la utilidad de la producción económica; a lo que habría que sumar espacios de investigación con otros criterios, a los cuales suele responder el ámbito público, el del Estado. En suma, "deben existir marcos de investigación públicos y privados, para que los investigadores encuentren posibilidades en unos u otros" (Ciuro Caldani 1992:854).

El gobierno de Fernández insistió en la vinculación de la ciencia con el sector productivo: "Nuestra hipótesis es que es posible que en la Argentina la inversión que la sociedad hace en Ciencia y Tecnología redunde en mejora de la calidad de vida de la población. [...] 'Vamos a apoyar la ciencia, pero no a la ciencia orientada por la curiosidad solamente [...] Queremos que realmente la ciencia y la tecnología tengan resultados concretos, que sean un motor de la economía, que resuelvan problemas sociales" (Barañao 2012:340).

Hay que aclarar que no se trata de una hipótesis científica, sino de una opción política, y como tal, podría preguntarse al pueblo como cuerpo electoral, como se lo hace en muchos lugares del mundo a través de las instituciones de democracia semi-directa. La mejora de la calidad de vida de la población es el objetivo, para el cual hay que instrumentar medios. Las últimas palabras del ministro le adjudican a la ciencia finalidades para las cuales no está capacitada, ya que tienen que ocuparse de la economía los economistas y de los problemas sociales los políticos, también. Esto me recuerda el fenómeno de la medicalización de la vida, cuando se le asignan a la medicina responsabilidades para las cuales no está preparada. Aquí se daría lo que se conoce como "cientifización de la vida". Esta confusión se ve en el ministro de Fernández cuando dice que la mayor demanda social de Argentina es la inclusión social (Barañao 2012:340), escribiendo en un artículo que se pregunta a dónde va la ciencia, cuando no es su tarea la inclusión social. No está aislada del mundo y muchos de sus campos apuntarán a resolver problemas sobre la inclusión social, pero su objetivo es alcanzar la verdad, o la información, como cuando el ministro habla de la tarea del doctor: "La sociedad le paga al investigador por tener información útil” (Barañao 2012:340).

Quedándonos en el ámbito de la planificación, cabe preguntarse si los objetivos o finalidades que tienen que llevar a cabo los organismos científicos deben ser planteados por ellos mismos o alguna suerte de "comunidad científica", o si tienen que provenir de los actores políticos, especialmente el Poder Ejecutivo. 
Hay que destacar la ausencia de agremiación, fundamental para luchar contra el poder. Agrupamiento que sería necesario actualmente, frente a algunos desaciertos del CONICET en cuanto a la precarización de los becarios como trabajadores informales, ya que terminada la beca o las becas, si fueron becarios doctorales o postdoctorales, y pasados más de 6 o 7 años aproximadamente, pueden no ingresar a carrera del investigador habiendo trabajado durante todo ese tiempo como meros "recursos humanos en formación". Siendo que en la realidad asistían a sus lugares de "trabajo", eran controlados por sus directores, informaban de sus avances, recibían dinero mensualmente y un suplemento por sus gastos médicos. A lo que hay que sumar la dedicación exclusiva que demandan las becas, es decir, el investigador no se puede dedicar a otro trabajo.

La información de prensa disponible muestra restricciones al ingreso a carrera del investigador, evaluaciones de ingresos no transparentes, falta de remuneración justa por los estudios realizados, sobre todo en comparación con otros sueldos de otros oficios o profesiones que no tienen necesidad de titulaciones. Cuando "en el año 2005 [...] a los 1500 nuevos becarios ingresados se le suman no menos de 500 investigadores en la carrera, proyectándose para el año siguiente la continuidad de la misma política de crecimiento" (Atrio 2006:67). Frente a este cuadro de situación "debe asegurarse la mayor estabilidad de los investigadores que sea compatible con sus áreas de labor, llegando en el marco público a una inamovilidad análoga a la de los magistrados judiciales" (Ciuro Caldani 1992:855). La exigencia de dedicación exclusiva (resolución 3224/2011 del Conicet) es auspiciosa en tanto permite un vuelco total de los esfuerzos en el trabajo científico, pero impide tener alternativas laborales en caso de cese de la actividad científica, como ocurría con las becas finalizadas y sin continuidad en el ingreso a carrera.

Durante mucho tiempo el Conicet ha hablado de la formación de recursos humanos, "expresión contable del campo de la economía y la producción industrial" (Hurtado 2010:228) y por la cual se pone hincapié, por ejemplo, en el carácter de beca que reciben sus investigadores, en lugar de hablar de su vocación y estilo de vida y tratarlos como "trabajadores": "el Dr. Charreau [...] [hizo] hincapié en la necesidad de seguir atentamente las políticas sobre ingresos y promociones, porque restringirlos sólo constituye una señal inequívoca de la baja prioridad que se otorga a la formación de recursos humanos y abre justificados interrogantes respecto al futuro de los jóvenes" (Atrio 2006:61).

El resaltado señala la vinculación entre política, economía y ciencia. Si de algo sirve la intervención del Estado en materia científica es para valorizar el trabajo del investigador, no tratándolo como una mera unidad económica, contable. En este sentido, en 2014, puede leerse esta frase en la propia página del organismo: "Desde hace más de 50 años, el Conicet contribuye sustancialmente al desarrollo socioeconómico de la República Argentina. Formando recursos humanos, generando y transfiriendo conocimiento científico-tecnológico, y fomentando su aplicación, el organismo produce y agrega valor a las actividades de la sociedad y mejora la calidad de vida de los ciudadanos" (Conicet 2014, el resaltado me pertenece). Lo que guarda relación con la opinión del gobierno de Fernández acerca de la política que debe seguir el país: "tienen recursos humanos, tienen un recurso que es renovable si se lo administra adecuadamente” (Barañao 2012:340, el resaltado es mío).

Habría aquí una "alienación científica", en tanto el Estado se queda con la vida de los profesionales que forma como científicos pero que luego rechaza por cuestiones presupuestarias o políticas, y en algunos casos académicas: “El problema a investigar debe permanecer preocupando durante el 'tiempo libre' del investigador, y esto tiende a impedirle otras actividades. De aquí la gran dificultad para reorientar su vida en caso de desempleo" (Ciuro Caldani 1992:852). 
Ya vimos la exigencia reglamentaria respecto de las becas a la dedicación exclusiva. La injusticia es más clara cuando se compara la situación con los que ganan un concurso docente universitario, que se mantienen en el cargo hasta la jubilación, agregándose solo como exigencia el informe periódico de la carrera docente.

Desde las más altas esferas del gobierno de 2007-2015 se considera al científico como una unidad contable: "yo estoy pensando, en tanto funcionario público, en términos de defender la inversión de la sociedad y no tanto en el destino individual de cada persona" (Barañao 2012:349). No desnaturalizar al doctorado, base académica de la investigación científica, y proteger al investigador de las arbitrariedades del Estado o la empresa, dan utilidad y sentido a la rama: "el Derecho de la Ciencia, que ha de atender a la especial problemática de la realización de la verdad y contiene, entre sus temas principales, las cuestiones especialmente significativas de la protección del investigador" (Ciuro Caldani 1992:852). "Así como el liberalismo político protege al gobernado contra el gobernante, ha de desarrollarse el liberalismo científico, orientado a amparar al científico en general y al investigador en especial contra los gobernantes" (1992:854). También apunta a "evitar las evaluaciones tendenciosas" (1992:855). Alguna vez se ha dicho que no existe "la" ciencia, sino los investigadores científicos. Ante aquella manifestación, "hay que amparar al investigador en la realización de la verdad contra la subversión de los valores jurídicos inferiores, como la conducción, el poder, la cooperación, la previsibilidad, la solidaridad, el orden, etc." (1992:853). Así como el bien común no puede perjudicar al individuo, tampoco "la ciencia" debe perjudicar al científico. "El investigador es un hombre con características especiales; la investigación se jerarquiza por el hombre y el hombre se jerarquiza por la investigación" (1992:853). Es más: "el Derecho de la Ciencia tiene un importante ingrediente de Derecho Público, pero urge evitar que ese despliegue publicista lo haga totalitario, desconociendo el bien particular del investigador y de los demás miembros de la sociedad. Los argumentos de bien común, que por su despliegue más 'ideal' son más fáciles de falsificar, suelen implicar graves amenazas para la tarea de investigación” (1992:853).

La actitud hostil del gobierno de la ex Presidente argentina Cristina Fernández hacia ciertos becarios que pretenden ingresar a la carrera del investigador también se confirma cuando el Conicet emplea las figuras del MASVALORDOC (www.masvalordoc.conicet.gov.ar) como una forma de paliar las expulsiones. El Ministro de Ciencia escribe que hay que producir en base a los requerimientos, como el de los anticuerpos monoclonales, alrededor de los cuales los tratamientos son costosos (Barañao 2012:345). Hay que recordar que fue el premio Nobel César Milstein quien los descubrió al hacer ciencia básica, desalentada por Fernández, y que al irse al Reino Unido, logró el reconocimiento que no tuvo en su país, además de patentar la idea y obtener rentas de su descubrimiento: "el presidente del Medical Research Council, al otorgarle a Milstein el premio Millenium de dicha institución, expresó: 'Ningún otro científico del Medical Research Council ha efectuado semejante contribución a la ciencia, salud y riqueza de Inglaterra'" (Hurtado 2010:228).

El Ministro de Ciencia de Fernández decía que aquel programa es una forma de "ubicar doctores" (Barañao 2012:347): "si no pensamos en una alternativa de inserción, lo que tenemos que pensar es que tal vez el número de doctores que tenemos tendría que ser bastante menor" (Barañao 2012:348). Nótese que también se resalta la política de repatriación de los científicos, que es afortunada, pero que debe replantearse ante tantos que ya viven en "la Patria" y que pretenden ingresar a la carrera del investigador: "Claramente me parece que es un tema que amerita discusión de todos los involucrados, porque esto también es un cambio de la cultura, hay una responsabilidad de todos. De ver qué hacemos con los recursos humanos que estamos capacitando" (Barañao 2012:348, el resaltado es mío). 
Pero mientras tanto siguen las restricciones y la responsabilidad principal es la de quien da las órdenes y ejecuta políticas que direcciona. Cuando en 2006 se decía: "Actualmente, la Carrera del Investigador Científico y Tecnológico del CONICET, destinada a favorecer la plena y permanente dedicación de los investigadores a la labor científica y tecnológica, está en un proceso de crecimiento continuo. Su actual administración ha conseguido eliminar los cupos para el ingreso, basando este proceso únicamente en el mérito de los postulantes" (Atrio 2006:84).

MASVALORDOC es un programa que apunta a insertar a los doctores en el sector productivo, industrial, de servicios, educación y gestión pública. En su lema dice: "sumando doctores al desarrollo de la Argentina", lo que encierra un error, ya que el doctor existe para investigar, básicamente, no para ejercer la técnica o hacer "desarrollo". De lo contrario se hablaría del tecnólogo. Esto ya ocurrió: "privilegió las 'aplicaciones científicas', se exaltó la tendencia 'profesionalista' y la ciencia pura fue considerada una pérdida de tiempo, porque la Argentina 'no necesita sabios sino hombres de acción', como pontificaba en 1896 el ingeniero Alcides Romagosa" (Orione 2008:74). Pareciera entonces que se confirma una suerte de eterno retorno: "Quizás hoy la condena no se apoye, como entonces [a Galileo], en la pretensión de santidad, sino en la utilidad y sea menos explícita, pero al fin puede ser incluso más efectiva, por ejemplo, privando a la investigación básica no inmediatamente aplicable de los recursos que le son imprescindibles" (Ciuro Caldani 1992:852).

Se insiste en el error acerca del doctorado cuando el propio Ministro señalaba: "así como se le paga a doctores para que vayan a empresas, se le va a pagar el sueldo durante dos años para que vaya a algún municipio a hacer algún trabajo" (Barañao 2012:347). Este desacierto es parecido al que existiría si a un docente se le plantea el trabajo de psicopedagogía, administración o gestión en la escuela. Puede hacerlo, pero no está capacitado para ello. El doctor investiga porque se formó para ello. Lo que no impide que aquellos que tengan la idea de una vinculación estrecha entre ciencia y desarrollo o economía o producción, la pongan en práctica. La página de MASVALORDOC se parece a los avisos clasificados de un diario, donde pueden leerse pedidos como estos: mejorador de maíz, ingeniero químico mecánico, especialista en tribología, especialista en cerámicas, especialista en diseño de cámaras de combustión, especialista en programación de microcontroladores para control de unidades, especialista en agronomía/biotecnología, gerente de cuentas para alimentos y bebidas, microbiólogo, director técnico con matrícula nacional, regulatory specialist, médico-salud ocupacional. Solo hay un pedido de investigador senior en química.

Así se anunciaba durante la presidencia de Charreau: "entre los principales logros se cuenta la creación de una nueva categoría de investigadores en empresas. Esto permitía insertar en el sistema productivo recursos humanos altamente calificados" (Atrio 2006:63, el resaltado es mío). Pero el científico o doctor no tiene por qué cumplir una labor que es del Estado: "la idea es que el municipio que tiene un doctor, tenga además de la marca de calidad, la posibilidad de acceder a financiamiento porque el propio doctor se supone que sabe escribir el pedido de subsidio y va a poder conseguir los fondos que muchos municipios no reciben porque no tienen a alguien capaz de escribir un proyecto" (Barañao 2012:347). Esto se parece a la estrategia del gobierno de Fernández de convertir a los consumidores en una suerte de guardia urbana que ayude a controlar los precios de los productos de los supermercados, cuando a los precios, o los controla el mercado, o los controla el gobierno.

Quien se presenta a la carrera del investigador científico suele haber pasado antes por la formación doctoral y posdoctoral, lo que sistemáticamente lo ha preparado para investigar y no ocurre de un día para el otro. Recordando la tesis weberiana según la cual el puritanismo ha influido en el capitalismo, y éste a 
su vez en la ciencia, se dice: "El puritanismo exigía un trabajo sistemático, metódico y una diligencia constante en cada uno" (Merton 2008:123). Cabe preguntarse por qué el Estado no tiene un funcionario capacitado para traducir las necesidades de una región en pedidos o soluciones. ¿No es esa la labor de un político como candidato o de un funcionario político ya electo o en funciones?

Ahora se entiende por qué son tan restrictivos los ingresos a la carrera del investigador. Porque una masa de doctores tiene que ir destinada al mercado, a la aplicación, a la técnica: "de esa cohorte de doctores, el CONICET selecciona aquellos que tienen habilidades para convertirse en investigadores científicos" (Barañao 2012:348). Y ahí es donde se ejerce la discreción, seleccionado aquellos doctores que serán "investigadores científicos", no porque tengan capacidad, sino porque la mano invisible del Estado o el padrinazgo dirá quienes tienen esa vocación por la ciencia. Como dice Barañao: "Tanto en EE.UU. como en Europa, el número de doctores que logra un puesto estable es mínimo" (2012:348). Lo que rememora a la "mano invisible" del mercado, tan criticada desde posiciones que se dicen progresistas. Lo que en el fondo encierra la mayor de las discrecionalidades. Aquí se ve la utilidad del pensamiento complejo: "La complejidad no es una totalidad cerrada, pero es aspiración a lograr algún grado de completud. De manera que aquello que es mutilado en un objeto disciplinar, tiene como consecuencia necesaria la manipulación antojadiza de aquello que es ocultado" (Galati 2009).

Esta política también se puede ver en una encuesta que envió en 2013 el Conicet a los doctores que formó llamada "consulta doctores 2013". Si se desea vincular la ciencia con la industria o la producción, podría encargarse dicha tarea a la industria, capacitada para innovar productivamente. El ministro de la presidente Fernández reconoció que hay que invertir en tecnología esperando resultados, pero no en ciencia esperando aplicaciones: "cómo esta inversión en conocimiento se acopla a la mejora de la inclusión social, creemos que apoyando el desarrollo de pequeñas y medianas empresas, de base tecnológica, es posible generar trabajo de calidad" (Barañao 2012:341). Por otra parte, el Ministerio es también de "innovación productiva". Desde allí mismo se reconoce "cómo acoplamos la ciencia innovadora [sic] a la actividad productiva. [...] Una [forma] es promoviendo empresas de base tecnológica" (Barañao 2012:344). O podría replantearse la titulación del doctorado, enseñando a los futuros doctores a aplicar conocimientos, en lugar de crearlos.

La aplicación luego del entrenamiento o adiestramiento es la labor del especialista, clase de posgrado que más afinidad tiene con las carreras de grado y la aplicación de conocimientos. Además, la iniciativa supone una red de vínculos entre el Conicet y las universidades, con la sociedad. Es decir, "políticas robustas de articulación de sectores" (Hurtado 2010:239). Todo lo cual es débil en Argentina: "la primera comunidad de investigación tecnológica se originará en Alemania, entre 1860 y 1880, que poseía una industria técnicamente avanzada en acelerado crecimiento, una universidad relativamente autónoma y una plétora de agencias de regulación y metrología locales y nacionales. [...] Este tipo de procesos de integración de componentes institucionales de regulación y metrología, universidades, instituciones públicas de investigación y desarrollo y firmas privadas promovido por una comunidad intersticial de productores de instrumentación genérica no tuvo lugar en la Argentina" (Hurtado 2010:238).

Un defecto que se percibe en el CONICET es la compartimentalización de la investigación científica al exigirse la ultra-especialidad: "En una evaluación de 2002, encargada por la revista Ciencia Hoy a un grupo de evaluadores internacionales, se lee: 'El comité advirtió la presencia de obstáculos burocráticos significativos, especialmente la poca permeabilidad de las fronteras que separan las ramas disciplinarias y la rigidez del sistema'" (Hurtado 2010:17). De hecho se sobrevalua la tecnificación o aplicación en lugar de la interdisciplinariedad. La hiper-especialización conspira contra los contactos, justamente de la 
investigación científica con la sociedad. Y porque los problemas no tienen que ver con una disciplina, sino que existen sin tener en cuenta lo que artificialmente el hombre ha creado pensando que por dividir se aborda mejor una problemática. No hay que dejar de resaltar el basamento cultural de la compartimentalización, en tanto ya ha calado hondo en la mentalidad de los administradores del conocimiento, como ciencia y enseñanza, y que se ve también en la universidad argentina, confederada en facultades. Un pensamiento de Ricardo Guibourg resulta desestructurante y mueve a pensar, cuando se refiere a la clasificación de las ciencias y al "objeto" de la ciencia. Señala que sabemos que la anatomía trata de la descripción del cuerpo, que la zoología se refiere a los animales, la astronomía a los cuerpos celestes y la economía a la producción y al intercambio de bienes. Pero entonces se pregunta, quién ha trazado esas divisiones. El hombre corta la pizza del conocimiento científico según sus intereses, sus creencias o sus prejuicios. Si los pajaritos tuvieran ciencias semejantes a las nuestras, no extrañaría que el ser humano formara parte de la zoología y la anatomía estaría reservada al cuerpo de las aves (Guibourg et.al. 2004).

En lo relativo a la organización y fundación de instituciones, en este caso las relativas a la ciencia y la investigación, como universidades y organismos específicos (CONICET y similares), la historia argentina previa a la reinstauración de la democracia en 1983, fue rupturista y no continuista o dialoguista. Así, "esta tensión, entre muchas otras, se iba a 'resolver' con un golpe de Estado y la cancelación, a contramano de lo que ocurría en los países avanzados, de uno de los polos de discordia" (Hurtado 2010:91). En relación a las causas de los fenómenos, y en este caso relativa al problema de la ciencia en Argentina, se ha dicho que: "El atraso que padecemos en nuestra conciencia de comunidad científica tiene raíces que deben buscarse en la influencia de determinantes psicosociales vinculados con la marginalidad social de nuestra ciencia y con el efecto de la represión política, que forjaron en nuestra comunidad una actitud anodina de riesgosa apuesta a una estrategia de autoconservación condicionada" (Orione 2008:82).

Como consecuencias de lo señalado en referencia a la compartimentalización e hiper-especialización, se señala: "sin los países en desarrollo actuando como proveedores de materias primas, lugar donde instalar los procesos industriales más contaminantes, donde realizar ensayos clínicos de medicamentos rápidos y económicos y, sobre todo, como pagadores de royalties" (Hurtado 2010:25). Estas consecuencias que impactan en el plano económico y político, luego repercuten en las planificaciones científicas o en la ausencia de ellas, en los países periféricos o en vías de desarrollo. En el marco de la ecología de la acción y previendo las consecuencias de nuestros actos, hay que pensar que es posible que ante una acción determinada se generen los resultados contrarios, no previstos por el autor: "Al crear el Conicet puso en marcha un factor que, contra sus esperanzas, iba a contribuir a la larga al deterioro de la investigación científica: el Conicet fue la presa codiciada de los sectores de ultraderecha y fuente de los más diversos negociados. Y en el Conicet nació un engendro que no hubiese podido ser parido por ninguna universidad: la crotoxina" (Orione 2008:36).

\section{Conclusión}

Es inevitable entonces lo que reconoce un propio funcionario que estuvo relacionado con el gobierno del CONICET: "sectores de la comunidad científica, que orientaban los recursos institucionales en su favor durante el período en que ejercían el poder de la institución" (Del Bello 2009:79). Se trataba entonces de "las tensiones generadas en un sistema que viene acumulando vicios desde hace años y que, en no pocos momentos, se vio sometido por diversas autoridades a prácticas que combinaron abundantes desaciertos técnicos con actos de corrupción" (Ciencia Hoy 1997:2). 
Síntoma de las indefiniciones y temores hacia la comunidad científica fueron los vaivenes institucionales: "creada la entidad que debía encargarse de la formulación de políticas [CONACYT], no existe indicio más claro de la debilidad de esta función a lo largo de las décadas siguientes que las sucesivas mutaciones y peregrinaciones ministeriales del organismo" (Hurtado 2010:221). Si bien una golondrina no hace verano, y crear un Ministerio de Ciencia no le asigna de por sí un lugar importante a la ciencia de un país, contribuye a tomar consciencia de su relevancia. Así como creer que invertir en salud mejora la calidad de vida de la población, hay que tomar consciencia que invertir en la verdad y en la ciencia, también hace lo propio con el progreso de un país.

Cabe recordar lo que en su momento dijo un ministro: "A mí me gustaría ver un cierto cambio metodológico en esas ciencias; estoy tan acostumbrado a la verificación empírica de lo que digo, que a veces los trabajos en ciencias sociales me parecen teología". También dijo que a veces son muy subjetivas. Es importante tener en cuenta esta suerte de "guía rápida para la elaboración de una comunidad científica" a los fines de sentar las bases filosóficas de una estrategia científica, para lo cual hay que cuidarse de "[los que] escribían en términos oscuros que parecían profundos; ridiculizaban la pretensión de los científicos de que el mundo puede describirse de manera objetiva; denunciaban a las ciencias sociales como imposibles y proclamaban la supremacía del espíritu sobre la materia, y la superioridad del sentimiento y la intuición por sobre la experiencia y la razón, al mismo tiempo que simpatizaban con los partidos de derecha y adulaban a los militares" (Orione 2008:40).

La gran respuesta a las preguntas se sintetiza en el papel del Estado en el desarrollo científico. La gran pauta puede estar dada por un diagnóstico de la situación, ya que no es la misma la situación científica en un país periférico que en un país calificado como potencia mundial. Hecha esa radiografía situacional, cabe reconocer a la ciencia como uno de los pilares básicos de los pueblos, para su dignidad como tales y para su desarrollo económico. En ese sentido, no puede ser tratada como una mercancía más sujeta a los vaivenes del mercado. Habría que lograr entonces una complementariedad entre las necesidades de la población y la autonomía de los investigadores en el diagrama de las políticas científicas. Así como el PAMI (Programa de Atención Médica Integral) tiene que ser de los jubilados, el CONICET tiene que ser de los científicos: "A partir de mayo de 1973 hasta el año 1981 el Consejo fue intervenido por los diferentes gobiernos que se sucedieron (civil en primer término y militar luego)" (Atrio 2006:46). Esto no significa aislarse de la vida pública ya que están en ella. Será tarea entonces lograr las vinculaciones entre el Estado y la sociedad civil con el organismo de ciencia argentino. La base de la complejidad es la comprensión de la diversidad en la unidad. Como dice Morín: "El control mutuo y la permutación de las jerarquías según las situaciones contrabalancean las tendencias parasitarias propias del mono-centrismo y el monojerarquismo" (2006:373). Así como en momentos de intervención se debe propender a la autonomía y viceversa, habrá que lograr dicha armonía a los fines de articular a la ciencia con la sociedad y a la sociedad con la ciencia. Esta articulación es labor del Estado y no de los científicos, que no gestionan la ciencia, sino que, fundamentalmente, la hacen.

Sería importante aspirar a que el Estado contribuya a la ciencia desde un organismo centralizado que marque pautas de desarrollo. También hay que destacar que la ciencia no se reduzca al Estado, ya que suele ser botín de los gobiernos que acceden al ejercicio del poder, no siempre vinculado con la verdad. La historia no solo nos ayuda a pensar la ciencia sino a tener en cuenta que está hecha por los científicos, que no dejan de ser hombres. Además, lo considerado normal y común en un período determinado, según una colectividad de especialistas en una institución determinada, puede ser siempre pensado de otra manera. 


\section{Bibliografía}

Abeledo, C. 2009. Ciencia y tecnología en el retorno a la democracia. En: M. Borches et.al. Ruptura y reconstrucción de la ciencia argentina. Buenos Aires: Dirección Nacional de Relaciones Internacionales, pp. 76-77.

http://www.mincyt.gob.ar/_post/descargar.php?idAdjuntoArchivo=22568

Adler, E. 1999. Si yo fuera presidente del Conicet. Medicina 59(1): 105-106.

http://www.medicinabuenosaires.com/revistas/vol59-99/1/v59_n1_105_106.pdf

Atrio, J. 2006. Conicet: ciencia y tecnología para el desarrollo. Buenos Aires: Edición Nacional. http://www.oei.es/salactsi/conicet.pdf

Barañao, L. 2012. ¿Hacia dónde va la ciencia argentina? Medicina 72(4): 339-349. http://ref.scielo.org/vvq7hy

Charreau, E. 2008. Conmemoración del quincuagésimo aniversario del CONICET. Medicina 68(3): 261-262.

http://ref.scielo.org/nb3tyv

Ciencia Hoy. 1997. La necesaria reforma del Conicet. Revista Ciencia Hoy 7(40):1

http://www.cienciahoy.org.ar/ch/hoy40/editorial.htm

Ciuro Caldani, M. 1992. Derecho de la ciencia y protección del investigador: hacia una constitución más humanista de la vida jurídica. Jurisprudencia Argentina 3: 851-857.

Del Bello, J. 2009. Contrareforma (1990/96) y cambios en el Conicet a partir de 1996. En: M. Borches et.al. Ruptura y reconstrucción de la ciencia argentina. Buenos Aires: Dirección Nacional de Relaciones Internacionales, pp. 78-80. http://www.mincyt.gob.ar/_post/descargar.php?idAdjuntoArchivo=22568

Dosne Pasqualini, C. 2008. El significado de la carrera del investigador del CONICET. Una visión personal. Medicina 68(4): 311-314. http://ref.scielo.org/hfs87j

Galati, E. 2009. La teoría trialista del mundo jurídico y el pensamiento complejo de Edgar Morin. Coincidencias y complementariedades de dos complejidades. Tesis doctoral. Rosario: Universidad Nacional de Rosario.

Guibourg, R. et.al. 2004. Introducción al conocimiento científico. Buenos Aires: Eudeba.

Hurtado, D. 2010. La ciencia argentina. Un proyecto inconcluso: 1930-2000. Buenos Aires: Edhasa.

Lorenzano, C. 1995. Cinco tesis para la historia de la ciencia. Actas de las Jornadas de Epistemología de las Ciencias Económicas. Buenos Aires: FEPAI. www.clorenzano.com.ar/Historia_files/cincotesis.pdf

Lorenzano, C. 2013. La institucionalización de la ciencia argentina. El pensamiento de Bernardo A. Houssay. Blog post. www.clorenzano.com.ar/Historia_files/houssay1.pdf

Merton, R. 2008. Le puritanisme, le piétisme et la science. En: J-F. Braunstein. L'Histoire des Sciences. Méthodes, styles et controverses. Paris: Vrin, pp. 119-146.

Morin, E. 2006. El Método 2. La vida de la vida. Madrid: Cátedra.

Morin, E. 2005. Introducción al pensamiento complejo. Barcelona: Gedisa.

Orione, J. 2008. Historia crítica de la ciencia argentina. Del proyecto de Sarmiento al reino del pensamiento mágico. Buenos Aires: Capital Intelectual.

Pérez-Tamayo, R. 2001. Ciencia básica y ciencia aplicada. Salud Pública de México 43(4): 368-372.

http://www.scielosp.org/pdf/spm/v43n4/5903.pdf

Recibido el 9 Oct 2015

Aceptado el 4 Ene 2016 